\title{
An Efficient Energy Aware Routing Mechanism for Wireless Body Area Networks
}

\author{
Wejdan Wasel Aljaghthami", Mohammad Haseeb Zafar and Afraa Zuhair Attiah
}

Faculty of Computing and Information Technology, King Abdulaziz University, Jeddah, 21589, Saudi Arabia

*Corresponding Author: Wejdan Wasel Aljaghthami. Email: waljahgthami@stu.kau.edu.sa

Received: 01 May 2021; Accepted: 01 June 2021

\begin{abstract}
The accelerated development of wireless network technology has resulted in the emergence of Wireless Body Area Network (WBAN), which is a technology commonly used in the medical field. WBAN consists of tiny sensor nodes that interconnect with each other and set in the human body to collect and transmit the patient data to the physician, to monitor the patients remotely. These nodes typically have limited battery energy that led to a shortage of network lifetime. Therefore, energy efficiency is considered one of the most demanding challenges in routing design for WBAN. Many proposed routing mechanisms in WBAN did not cover the source node energy and energy harvesting techniques. Therefore, this study proposes an Efficient Energy Aware Routing (EEAR) mechanism. This paper constructs a path cost function that considers three parameters: residual energy, number of hops to the sink, and the distance between the nodes. Besides, data aggregation with filtration and hybrid energy harvesting technique are used to extend the network lifetime, reduce the network traffic load, and maintain the source node energy. Extensive simulations using MATLAB have been performed to evaluate the performance of the proposed mechanism. EEAR is contrasted with the two latest schemes, called Priority-based Congestion-avoidance Routing Protocol (PCRP) and Energy Efficient Routing Protocol (EERP). The results show the significant performance of the EEAR mechanism in terms of network lifetime, residual energy, network stability, and throughput.
\end{abstract}

Keywords: Wireless body area network; routing protocol; energy efficiency; network lifetime; energy harvesting

\section{Introduction}

In the midst of the rapid growth of sensors and wireless communications technology, Wireless Body Area Network (WBAN) has emerged as a new concept. WBAN is a network consists of sensors that have different energy, sizes, demands, functions, etc. They can be wearable or embedded inside the body used for monitoring the human body to support a variety of applications (for example, military, medical, and sports) [1-3].

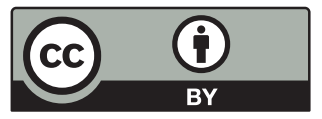

This work is licensed under a Creative Commons Attribution 4.0 International License, which permits unrestricted use, distribution, and reproduction in any medium, provided the original work is properly cited. 
The communication architecture in WBAN is separated into three tiers, as shown in Fig. 1.

- The tier-1 (Intra-WBAN) consists of different Sensor Nodes (SNs) that communicate with the sink via a wireless medium. A sink is a device that has a large capability in processing and storing data, which use to collect data from SNs then transmit it to the gateway [4].

- The tier-2 (Inter-WBAN) consists of mediator devices like personal computers that send data to the remote site.

- The tier-3 consists of remote servers that supply users by different applications [2,5].

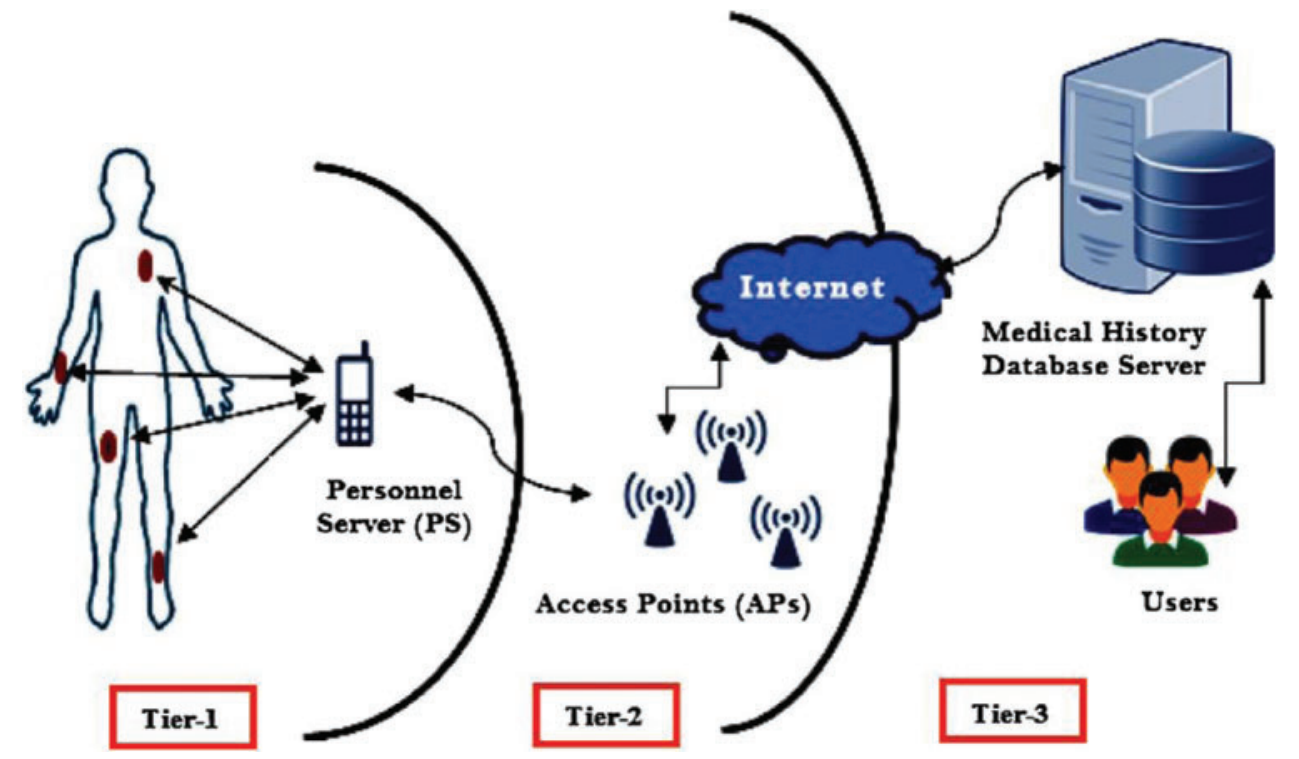

Figure 1: The structure of communication in WBAN [1]

WBAN has many applications in healthcare because of the advantages it offers. The physicians can monitor the medical situation of patients automatically and issuing reports about them periodically. The WBAN is also able to work on monitoring patients for a long period without impacting their daily activities. The patients that suffer from chronic diseases and elderly people can be monitored for their medical situation in their residences away from the hospital, which reduces the medicine cost and repeated visits to the clinics [2,6].

Despite the attractive features of WBAN, there are many challenges in the design of routing mechanisms, in which energy efficiency is considered one of the most demanding challenges in routing design for WBAN. Recently, the urgent need has been increased to use the SNs of the WBANs from the medical side to monitor the patients especially those having a chronic illness, infectious diseases such as the coronavirus (COVID-19), and older people [2]. Fig. 2 shows the examples of WBAN usage in the medical side.

Typically, these SNs depend on the battery as a source of energy which is limited and cannot be recharged, also some of the SNs are embedded inside the human body by surgery that becomes difficult to replace. Moreover, the deployment of SNs will impact their capabilities since the repeated use of the same node through the routing process will cause node energy consumption. In order to address these issues, researchers have proposed multiple routing mechanisms for 
optimizing energy resources routing and network topology of WBANs, but none of them provide sufficient solutions. The most used technique for the power supply is energy harvesting, but it is not enough to be energy efficient. It should exploit the energy of the source node in the power supply. Efficient routing can lead to reduced consumption of energy resources and increased network lifetime [1-3]. In order to overcome the limitations in the existing mechanisms, this paper presents an Efficient Energy Aware Routing (EEAR) mechanism. The contribution of this work is threefold: first, it constructs path selection function that considers residual energy, number of hops to the sink, and distance between the nodes. Second, it develops the aggregation and filtration mechanism to maintain the source node energy and balance network traffic load. Third, it implements a hybrid harvesting technique to recharge the SNs and provide sustainable energy addition to prolong network lifetime.

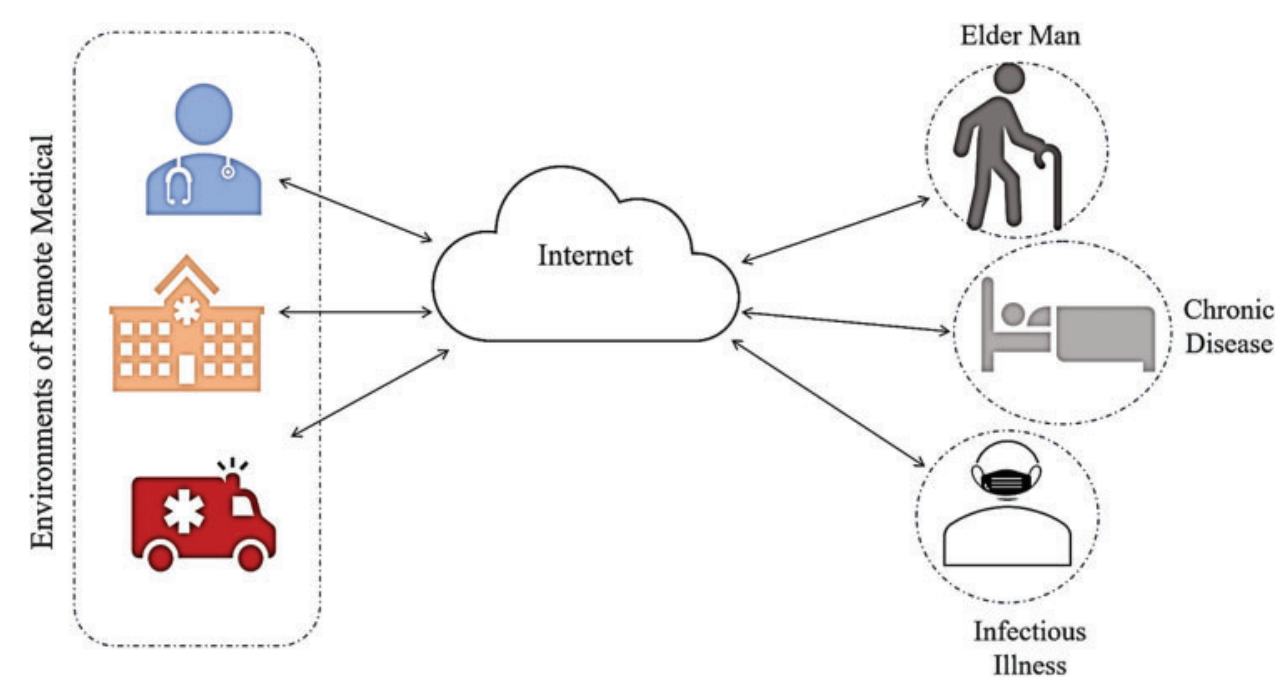

Figure 2: WBAN usage examples in the medical side

The rest of the paper is organized as follows. Section 2 provides details of related work, Section 3 demonstrates the system model, Section 4 presents the work principal of the proposed mechanism, Section 5 discusses the results and evaluations, and Section 6 concludes the paper and provides future work enhancements.

\section{Related Work}

We extensively looked for the mechanisms that overcome the issues mentioned in Section 1. Several proposed mechanisms apply various methods that attempted to keep up with the recent routing design requirements. Ullah et al. [7] proposed Energy efficient Harvested Aware clustering and cooperative Routing Protocol (E-HARP) for WBAN. E-HARP consists of fourteen heterogeneous sensor nodes on the human body alongside with two sink nodes. This protocol depends on the dynamic selection of $\mathrm{CH}$ and cooperative effort in routing. The optimum $\mathrm{SN}$ is selected as $\mathrm{CH}$ among the other SNs known as cluster members. The selection of $\mathrm{CH}$ depends on the cost factor that results from various parameters includes required transmission power, residual energy of SN, total network energy loss, and communication link Signal-to-Noise Ratio (SNR). Then, the protocol routes data by assisting from the SN by preventing redundant data from transmitting to maintain the energy of source nodes. E-HARP overcomes challenges of dynamic 
selection of $\mathrm{CH}$ and node energy consumption. However, employment of double sink needs extra network cost and the strategy of saving data packets in SN in order to prevent redundant data that may consume its memory [8].

Umare et al. [9] proposed a scheme for optimizing the clustering-based routing protocol using the Genetic Approach (GA). GA simulates the natural electing process and the evolution of biologically inspired by Darwinian theory. They used it to exist the optimum cluster head with considering the distance and residual energy of nodes.

Ullah et al. [10] proposed traffic priority-based delay-aware and energy efficient path allocation routing protocol for WBAN (Tripe-EEC). A data in this protocol is classified into four classifications, which include data on-demand, general data, critical data of high and low threshold values; each classification is treated in the special threshold according to residual energy and heat of sensor.

Awan et al. [11] proposed a multi-hop Priority-based Congestion-avoidance Routing Protocol for efficient energy in WBAN (PCRP). The data transmission in this protocol is classified into general and critical data. In critical data, two parameters are used to select the next hop: SNR and residual energy. In general data, they used two above parameters and congestion on-forwarder node (NCL) to select the next path. Besides, the technique of aggregation and filtration is used for the data inside the source node to minimize energy consumption and traffic load of the network.

Qu et al. [12] proposed an Energy Efficient Routing Protocol (EERP) for reliable data transmission in a WBAN. This protocol employed two or multiple hop communication to reduce the energy-consuming resulted from direct communication. They created the maximum Benefit Function $(\mathrm{BF})$, which normalizes various node parameters including transmission efficiency, number of hops to the sink, available bandwidth, and the residual energy. In order to choose the optimum next-hop node dynamically, which has the largest value and set the weight of BF dynamically depending on data priority. Similarly, an energy efficient routing protocol for Wireless Body Area Sensor Networks was proposed by Khan et al. [13]. This protocol includes eight SNs: two of them utilize to send emergency data to sink. Other SNs are utilized multiple hops for sending general data sensors. These SNs are considered as forwarder nodes, which compute node residual energy and distance from the sink. The two proposed protocols improved the network's lifetime and stability.

Anwar et al. [14] proposed Energy aware Link efficient Routing approach (ELR-W). This protocol includes eight SNs and one sink node. A new path cost function is constructed that considers multiple parameters including the link quality, distance to the sink, residual energy of node, and the number of hops. The weight elements of these parameters are predefined to specify the priority to every parameter when selecting the next hop.

Kumar et al. [15] proposed Energy Aware Power Save Mode Link State protocol (EAPSMLS). They adapted the current optimized link-state routing protocol by combining it with the power save mode. In this scheme, if the node has enough energy with second hop communication, then it is considered as a forwarder node; otherwise, it is handled as light or deep sleep mode according to its little remaining energy.

Amin et al. [16] proposed an energy-efficient model and path loss scheme (PLEEM). In this scheme, when the SN is placed near the sink, it directs the packet to the sink node. Otherwise, the SN transmits the packet by using the multi-hops approach. The forwarder node is selected based on the highest residual energy and least distance to the sink. 
Khan et al. [17] proposed an Energy Harvested and Cooperative Enabled Efficient Routing Protocol (EHCRP). This mechanism deploys eight sensors and two sink nodes overall body. The next path is selected according to various parameters: RE, SNR, NCL, distance to sink, and the number of hops count from the sink. Then, the mechanism routes data by assisting from the SN by preventing redundant data from transmitting to maintain the energy of source nodes. Furthermore, an energy harvesting technique is used to provide more energy and extend the network lifetime. EHCRP enhances energy efficiency and extends the network lifetime. However, employment of double sink needs extra network cost and the strategy of saving data packets in $\mathrm{SN}$ in order to prevent redundant data that may consume its memory [8].

Referring to the previous studies of the WBANs routing mechanisms, some of them did not take into account the priority in their routing data, while the other mechanisms directly transmit the emergency data that leads to consuming more energy when the nodes are located away from the sink. Most of the previous studies did not implement any mechanism for maintaining the source node energy which dissipates its energy in useless data and they lack the energy harvesting techniques to enable recharging the SNs battery and ensure sustainable energy. Therefore, we propose an energy-efficient stable mechanism, that leads to prolonging the network lifetime of nodes in the WBANs. The details of the mechanism are provided in Sections 3 and 4.

\section{System Model}

The system model of our mechanism is categorized into network model and energy model. More details are described in the following.

\subsection{Network Model}

The network topology of our scheme consists of one sink node situated on the body waist and ten SNs which are deployed over the human body as shown in Fig. 3 and Tab. 1.

In Fig. 3, the network topology includes two paths: direct path and routing path. The SNs closer to the sink will be connected through the direct path, while the farthest sensors through the routing path. These SNs send or forward the data toward the sink node. Moreover, each SN specifies its neighboring nodes by specific transmission range.

Our scheme follows the assumptions below:

- All SNs are deployed in the fixed position over the human body.

- All SNs have the same initial energy and processing power.

- The sink node has strong processing power, while limited in the SNs.

- Each $\mathrm{SN}$ is equipped with the hybrid energy harvesting technique.

\subsection{Energy Model}

Typically, the SNs have to sense, send, and receive the data over the WBAN; all these functions consume SNs energy. Our scheme focuses on the nodes' communications that consume energy in transmitting, receiving, and aggregating the data. We use the proposed energy model that is used in [18] The energy consumption model is described in the Eqs. (1) and (2).

$E_{t}=E_{t c} \times k+E_{a m p} \times D^{2}$ 
whereas $E_{t}$ denotes the transmitting energy in one sensor by transmitting $k$ bits data to another sensor at distance $D$. $E_{t c}$ and $E_{a m p}$ indicate the energy consuming by a circuit to transmit and amplify the data, respectively.

$E_{r}=E_{r c} \times k$

whereas $E_{r}$ denotes the receiving energy in one sensor by receiving $k$ bits picket size from other sensors.

In the WBANs, the loss coefficient $(n)$ for the human body in contrast with the Wireless Sensor Networks (WSNs) is different, so the equation Eq. (1) is rewritten in Eq. (3) [8].

$E_{t}=E_{t c} \times k+E_{a m p} \times D^{n}$

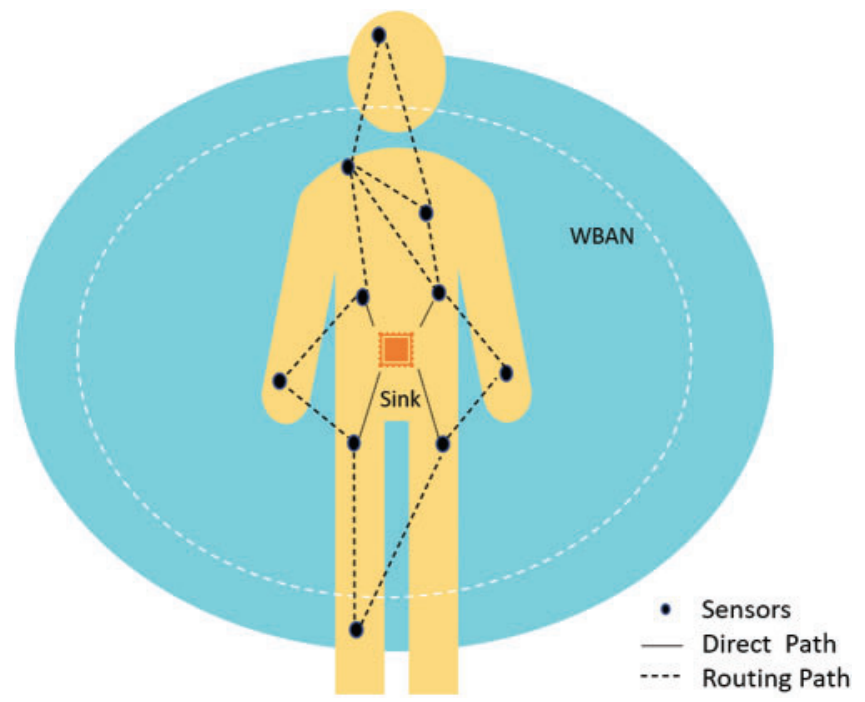

Figure 3: Deployment of the nodes in EEAR

Table 1: Location of the nodes in EEAR [17]

\begin{tabular}{llll}
\hline Node & Position on the body [17] & X position $(\mathrm{m})$ & Y position $(\mathrm{m})$ \\
\hline S1 & Head & 1.1 & 1.85 \\
S2 & Right Shoulder & 0.9 & 1.55 \\
S3 & Left Chest & 1.25 & 1.35 \\
S4 & Right Abdomen & 0.8 & 1.15 \\
S5 & Left Abdomen & 1.35 & 1.15 \\
S6 & Right Arm & 0.3 & 0.9 \\
S7 & Left Arm & 1.75 & 0.9 \\
S8 & Left Hip & 1.2 & 0.7 \\
S9 & Right U Hip & 0.8 & 0.7 \\
S10 & Right Foot & 0.8 & 0.2 \\
Sink & Waist & 1 & 1 \\
\hline
\end{tabular}




\section{Efficient Energy Aware Routing Mechanism}

Energy Efficient Aware Routing (EEAR) mechanism aims to alleviate the energy dissipated in the direct communication by adopting two-hops to three hops. Also, we construct a Path Selection Function (PSF) to enable a given sensor to dynamically selects the optimum path among the neighbors' nodes. PSF depends on three parameters: Residual Energy (Re), Number of Hops to the sink ( $\mathrm{NoH})$, and the Distance (D) between the given node and the neighbors' nodes. So, the node with the highest Re, lowest $\mathrm{NoH}$, and shortest $\mathrm{D}$ will be selected among the neighboring nodes. The same steps continue till the data reaches the sink node. Our scheme considers the priority of the data, whereas the emergency data will take the higher priority and direct transmission via an optimum path, while the general data will be aggregated for a period of time. In order to reduce the network traffic load and energy consumption, the aggregation and filtration technique for general data is used, which is adopted from [11]. We also use the hybrid harvesting technique proposed by Saraereh et al. [19] to cater limited battery energy issue. This technique harvest energy from two different sources Radio Frequency (RF) and thermal energy. The power generated from RF harvester at $2.4 \mathrm{GHz}$ around $0.740 \mathrm{~J}$ and from the thermal energy around $0.530 \mathrm{~J}$.

The EEAR consists of three phases: initialization phase, path selection phase, and routing phase. In this mechanism, the data will be processed and passed through these phases. The working principle for the EEAR mechanism is demonstrated in Algorithm 1 and more details are described below with the flow chart represented in Fig. 4. The three phases are described in the following.

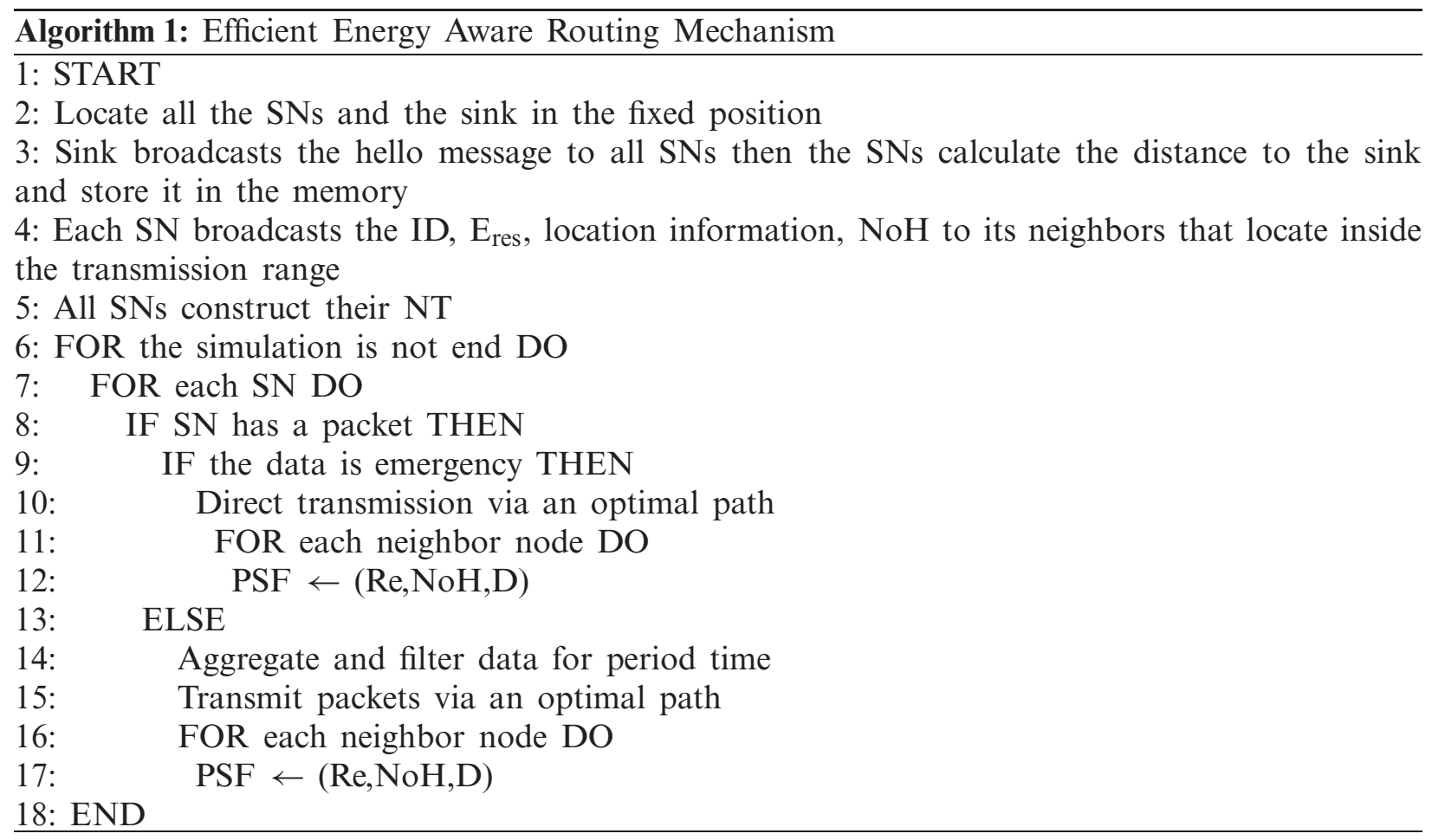




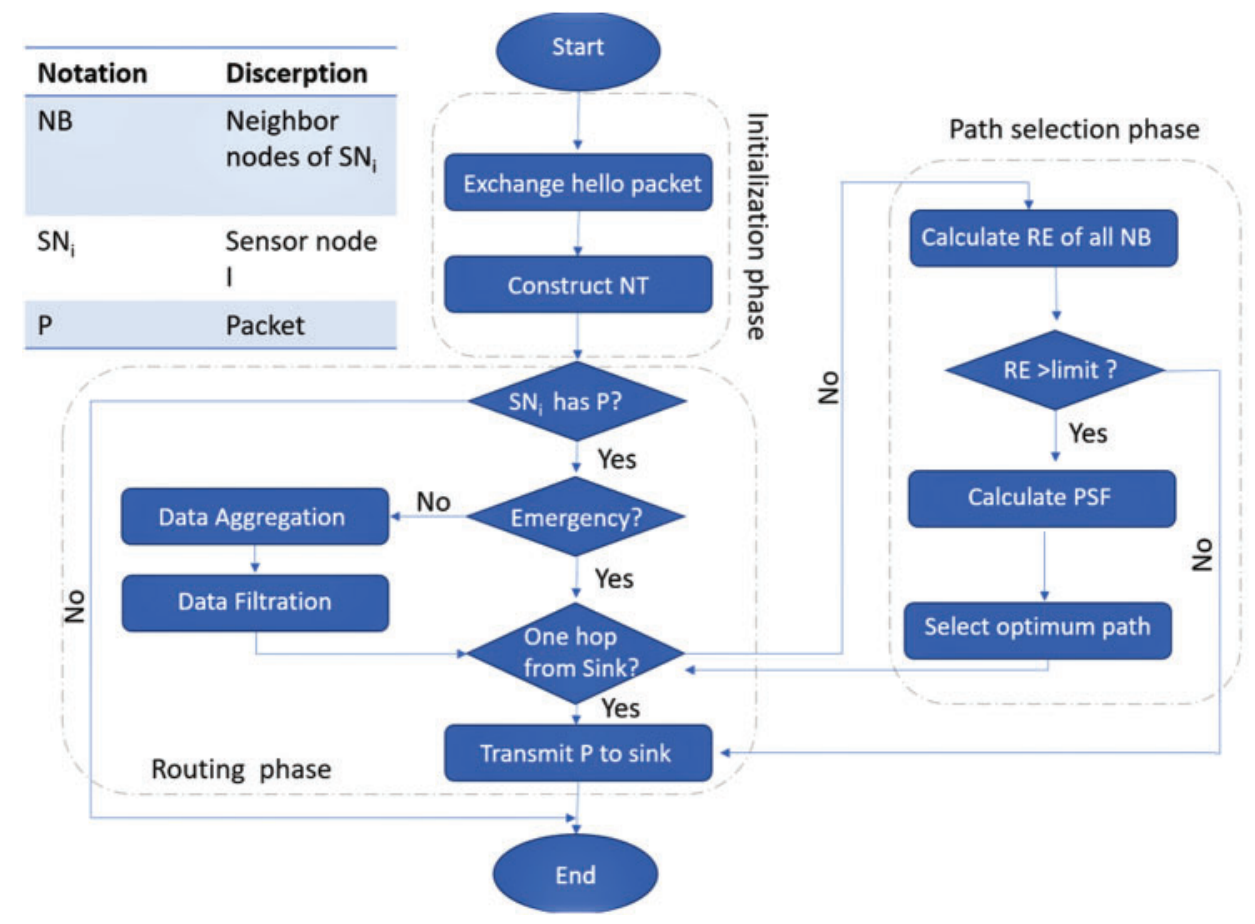

Figure 4: Flowchart of EEAR

\subsection{Initialization Phase}

In this phase, the SNs exchange hello packet with each other and construct the Neighbor Table (NT), the processes are described as follows. The sink node sends the hello packet to all SNs in WBAN, which includes its ID, and location information. The SNs receive this packet and update the sink location. The location of the sensor from the sink and the distance between communication parties are calculated by the basic distance Eq. (4).

$D=\sqrt{\left(X_{S}-X_{r}\right)^{2}+\left(Y_{S}-Y_{r}\right)^{2}}$

whereas the $\left(X_{S}, Y_{S}\right)$ and $\left(X_{r}, Y_{r}\right)$ represent the sender and receiver location, respectively.

Each SN sends the hello packet to all neighbors' nodes, which contains sensor ID, Re, NoH, and location information. At this moment, each SN can construct its NT.

\subsection{Path Selection Phase}

For the objective of enhancing the routing efficiency, we construct the Path Selection Function (PSF), which considers three parameters: Re, NoH, and D. The PSF is formulated in Eq. (5).

$P S F=\alpha \times R e+\beta \times \frac{1}{N o H}+\gamma \times(1-D)$

where $\alpha, \beta$, and $\gamma$ indicate the weight factors of the $\mathrm{Re}, \mathrm{NoH}$, and $\mathrm{D}$ respectively. The weights values are subject to:

$\alpha+\beta+\gamma=1$, they are assigned in the following values: $\alpha=0.5, \beta=0.3$, and $\gamma=0.2$. 
We normalize the Re parameter by applying min-max normalizing as formulated in the Eq. (6).

$R e=\frac{E_{\text {res }}-E_{\text {min }}}{E_{\text {int }}-E_{\text {min }}}$

where $E_{\text {res }}$ indicates the residual energy of the node before normalization, $E_{\min }$ is the value of minimum energy which equal 0 , and the $E_{\text {init }}$ refers to initial energy. The algorithm for the next path selection function is demonstrated in Algorithm 2.

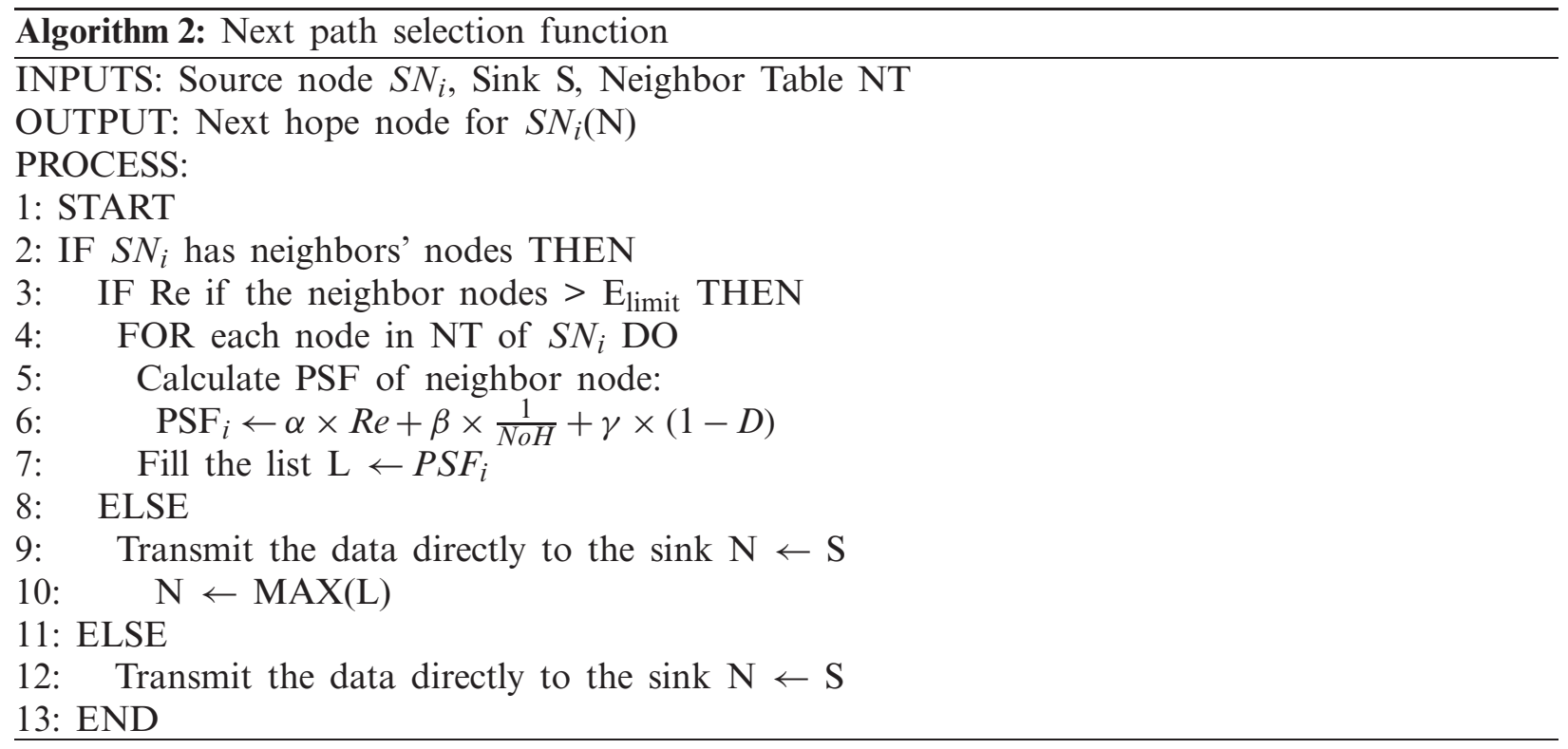

\subsection{Routing Phase}

In our scheme, the communication is carried out depending on the multi-hop communication. Thus, the SN that locates two or three hops from the sink will forward the packet via an optimum path (that is selected from its NT) till it reaches the sink. If the residual energy of all SN neighboring nodes is below the threshold, the SN will transmit the packet directly to the sink otherwise, keep forwarding.

In this phase, if the $\mathrm{SN}$ has a packet to send, it will forward it based on data priority. If the data is the emergency data, then it will be forwarded immediately through an optimum path by using Eq. (5) until it reaches the sink. Otherwise, the data will be aggregated and filtered based on the five categories rather than three such as in [11]. In order to achieve more data accuracy, for instance, the blood pressure consists of seven categories; five categories among them are considered as the general data [20]. Then, the data will be saved in the SN buffer for a limited period to reduce the redundant packets to maintain the source node energy and balance the load of network traffic. Afterward, the aggregated packets will be forwarded through an optimum path by using equation Eq. (5) until it reaches the sink. 


\section{Implementation and Evaluation}

A number of experiments using the MATLAB platform are performed to evaluate the performance of the EEAR mechanism. In the simulation, the number of rounds is specified as the time value. In addition to the occurrence rate of sending the packets and their priority is dependent upon the probabilities. Whereas the probabilities of the SNs that do not have a packet to send and in this regard the emergency data is taken 0.1 , otherwise is taken 0.9 times. The simulation parameters of Nordic nRF2401 [21] are used, because it has a single-chip transceiver that consumes the lowest energy among the other transceivers of the WBANs [8]. The metrics values are shown in Tab. 2.

Furthermore, five simulations were performed in each run, and the average of the metrics results are taken. The EEAR is compared with PCRP and EERP in terms of network lifetime, network stability, residual energy, and the throughput. PCRP is selected because it considers the source energy of the SNs and gives priority to the sensed data. EERP is similar to the proposed mechanism in the routing protocol classification and considers the same two parameters Re and $\mathrm{NoH}$ during the selection of a next hop. Furthermore, it gives priority to the sensed data. More details of those metrics with results and discussion are described as follows.

Table 2: Simulation parameters

\begin{tabular}{lll}
\hline Parameter & Value & Description \\
\hline Simulation Area & $2 \times 2 \mathrm{~m}^{2}$ & Area of topology (static) \\
Simulation Time & $1500 \mathrm{~seconds}$ & Approx. 25 min simulations \\
Number of SNs & 10 & Number of Sensor Nodes \\
Number of Sink & 1 & Only one sink node is used \\
$\mathrm{E}_{\text {init }}$ & $0.5 \mathrm{~J}$ & Initial Energy \\
$\mathrm{E}_{\mathrm{t}}$ & $16.7 \mathrm{~nJ} / \mathrm{bit}$ & Energy consumption for data transmission \\
$\mathrm{E}_{\mathrm{r}}$ & $36.1 \mathrm{~nJ} / \mathrm{bit}$ & Energy consumption for data receiving \\
$\mathrm{E}_{\text {amp }}$ & $1.97 \mathrm{~nJ} / \mathrm{bit}$ & Energy consumption for data amplifying \\
$\mathrm{PS}$ & $4000 \mathrm{bits}$ & The data packet size \\
Transmission Range & $1 \mathrm{~m}$ & The range of SNs transmission (static) \\
\hline
\end{tabular}

\subsection{Network Lifetime}

The network lifetime is defined as the total period that begins from running the network till the death of all SNs. This metric is considered one of the important characteristics of various sensor networks. The network lifetime is formulated in the Eq. (7).

Network lifetime $=\sum_{R=1}^{D N_{s}} R_{D N}$

where $R$ indicates the number of network rounds, $D N_{S}$ is the number of the death of all $\mathrm{SNs}$, and $R_{D N}$ the round of the dead SNs. The results of the network lifetime for the EEAR are compared with PCRP and EERP according to dead SNs. 
Fig. 5 shows the analysis of network lifetime. The results show that in EERP, the five SNs died at 2000 rounds. The SNs in EERP died early because of an increase in the network traffic load generated from transmitting the frequented data in each round. In PCRP, the first node died at 10750 rounds, while the first node of EEAR died at 11950 rounds. The entire nodes died at 7658 and 12110 rounds in the EERP and PCRP respectively, while the nodes of EEAR were still alive until 15930 rounds. Fig. 5 revers that the network lifetime of the EEAR mechanism is $106.6 \%$ and $31.5 \%$ longer than EERP and PCRP, respectively. EEAR has the longest network lifetime among others due to the SNs are equipped with energy harvesting technique. The second reason is to consider of the hops count during selecting the next hop node.

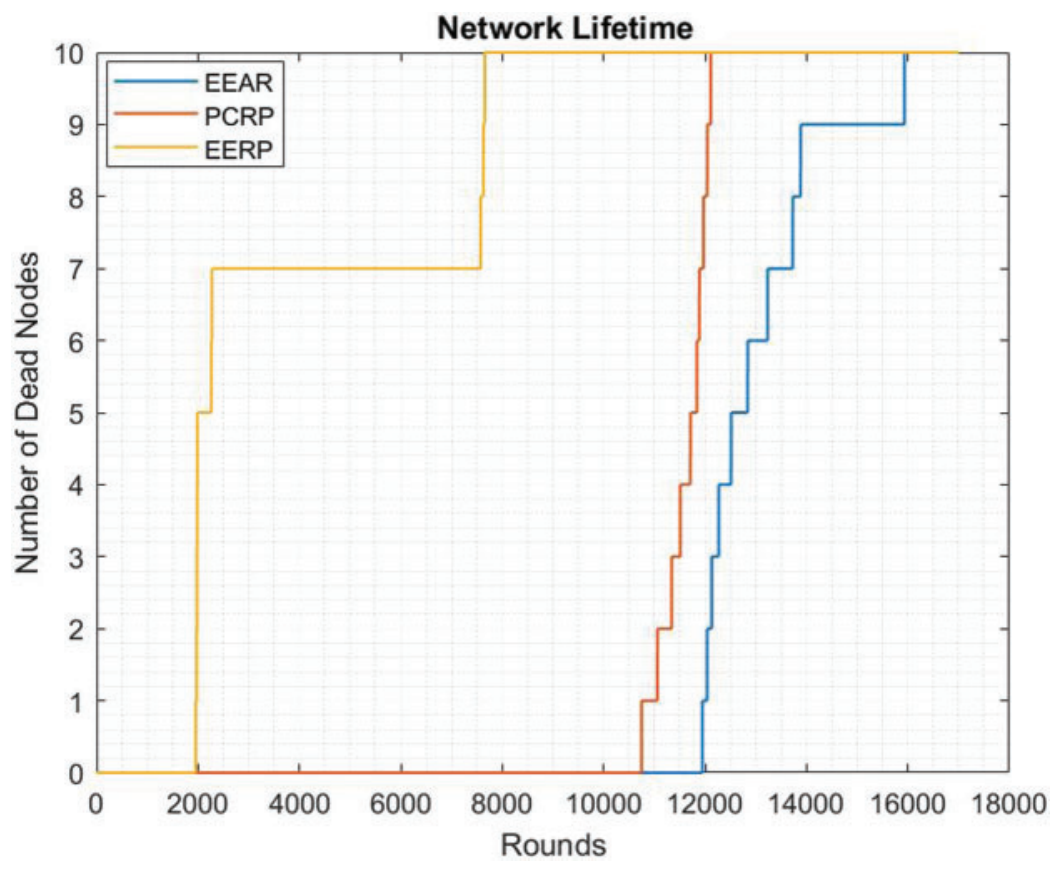

Figure 5: Analysis of network lifetime

Fig. 6 represents another view of the network lifetime in terms of first, half, and last dead SNs. We notice that the EEAR has the longest network lifetime among other mechanisms.

\subsection{Network Stability}

The network stability is defined as the total period that begins from running the network till the death of the first node or all SNs stay active. This metric is also considered as one of the important characteristics of various sensor networks. Network stability is formulated in the Eq. (8).

Network stability $=\sum_{R=1}^{N-1} R_{N-1}$

whereas $N$ indicates the number of SNs, and $R_{N-1}$ is the round of the first dead SN. The results of network stability for the EEAR are compared with PCRP and EERP in terms of the first dead node. Fig. 7 exhibits the analysis of network stability. The results show that the first node died at 
1956 rounds in the EERP. We notice that the first SN in EERP is dead quickly due to the heavy load traffic on the nodes that closer to the sink by transmitting frequented data in each round. The PCRP is stable until 10750 rounds, while EEAR until 11950 rounds. This attests the EEAR is $11.16 \%$ more stable than PPCR. Finally, the results show that the stability period of EEAR is the higher in contrast to EERP and PCRP due to applying the energy harvesting technique and considers the hops count parameter in the selection of the next hop node.

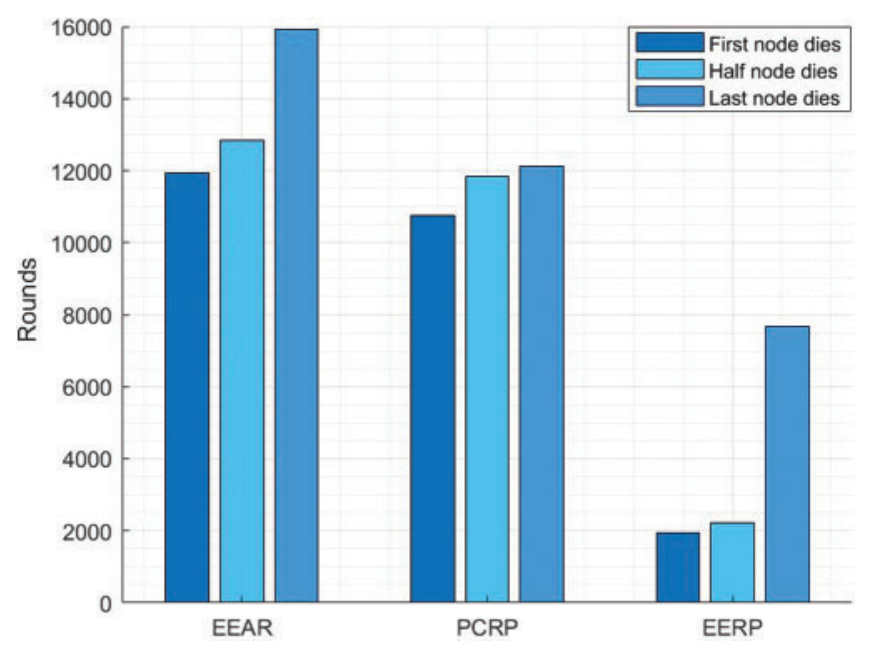

Figure 6: Analysis of network lifetime

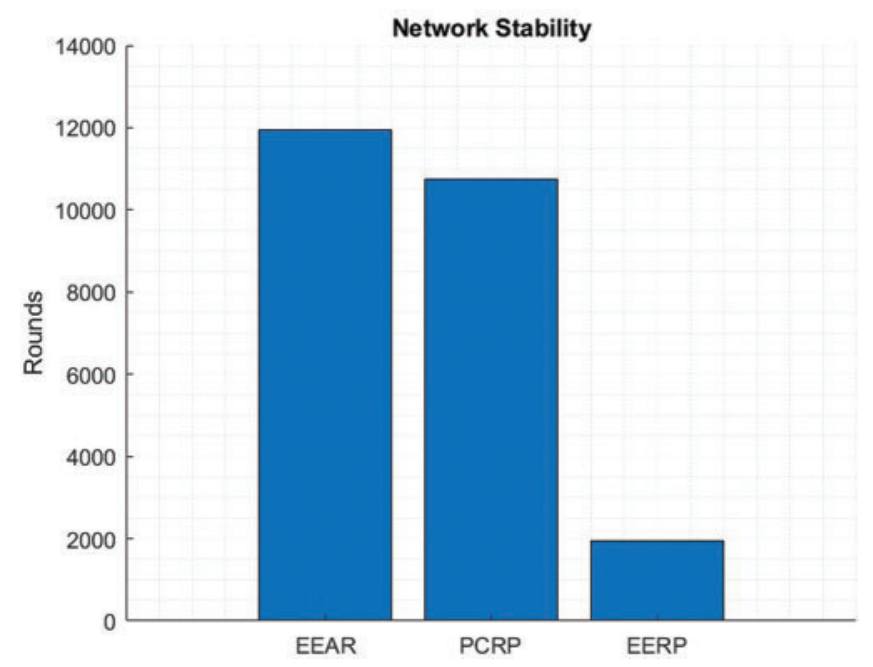

Figure 7: Analysis of network stability

\subsection{Residual Energy}

The remaining energy of the SNs in each round is called Residual Energy (RE) whereas the sum of the whole energy of SNs subtracts the whole energy consumption of SNs in each round. The results of RE for the EEAR are compared with the PCRP and EERP in terms of RE. 
The analysis of residual energy is shown in Fig. 8. We notice that the EERP energy is dissipated early and completely exhausted before 8000 rounds. In PCRP and EEAR, the remaining energy is approximately equal in ratio. In the round 8000th, the SNs battery of EEAP is recharged that reinforces the SNs performance. At the same time, more energy is dissipated in PCRP due to the adoption of only the two-hops count, that leads to consuming the energy of farthest nodes. In the last round, the SNs in EEAR still have energy and did not reach to the zero which is proved the efficient energy for the proposed mechanism.

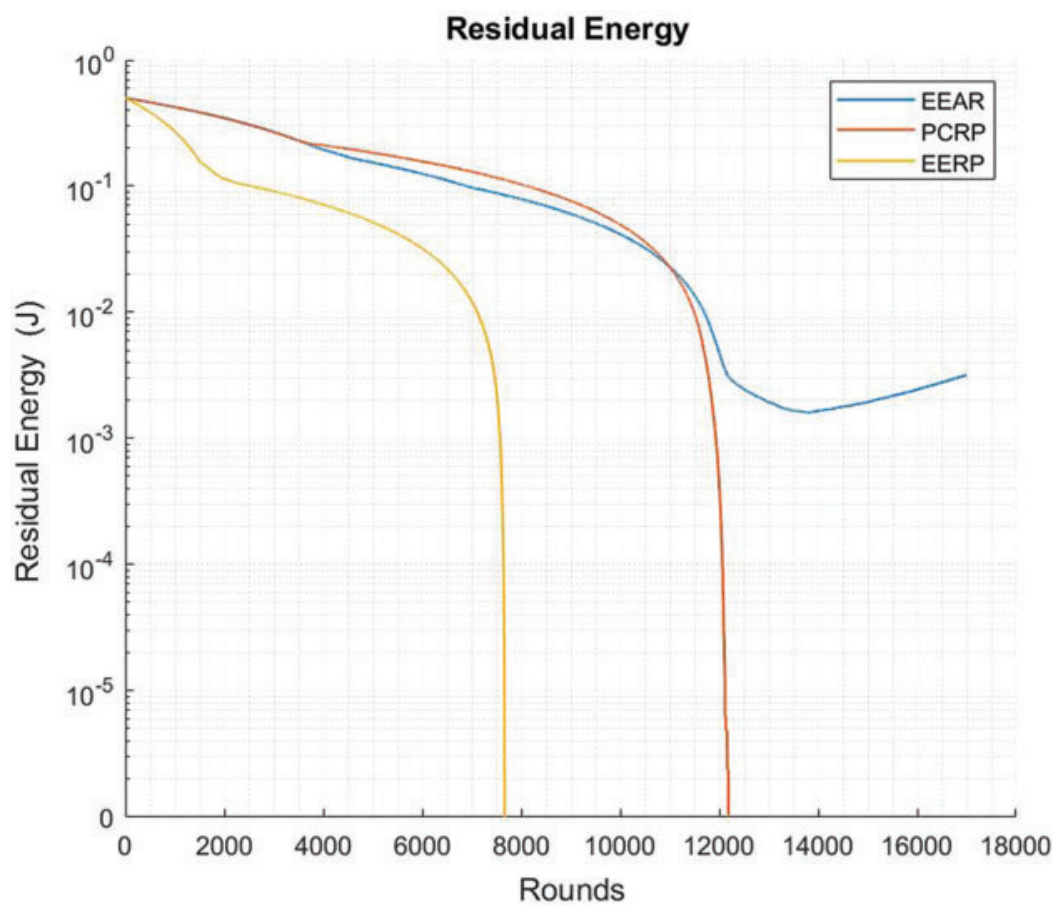

Figure 8: Analysis of residual energy

\subsection{Throughput}

The throughput is defined as the amount of the successful number received packets (RPs) from SNs to the sink in a specified time. It is considered as one of the main parameters of any WSN, high throughput realizes high network performance. Throughput is formulated in the Eq. (8).

Throughput $=\sum_{R=1}^{R_{\max }} R P s_{r}$

where $R P s_{r}$ is an accumulative number of successfully received packets at the sink in each round. The results of throughput for the EEAR are compared with PCRP and EERP in terms of the RPs at the sink.

Fig. 9 shows the analysis of throughput. The RPs of EERP reach 34270 packets. In the PCRP, the RPs reach 27150 packets, while reach 39260 packets in the EEAR. We notice that in the beginning rounds, the EERP receives the highest packets among the others due to 
transmit frequented data. In the ending rounds, the EEAR achieves more throughput among other mechanisms. The reasons are that the EEAR is the most stable and the longest network lifetime than others.

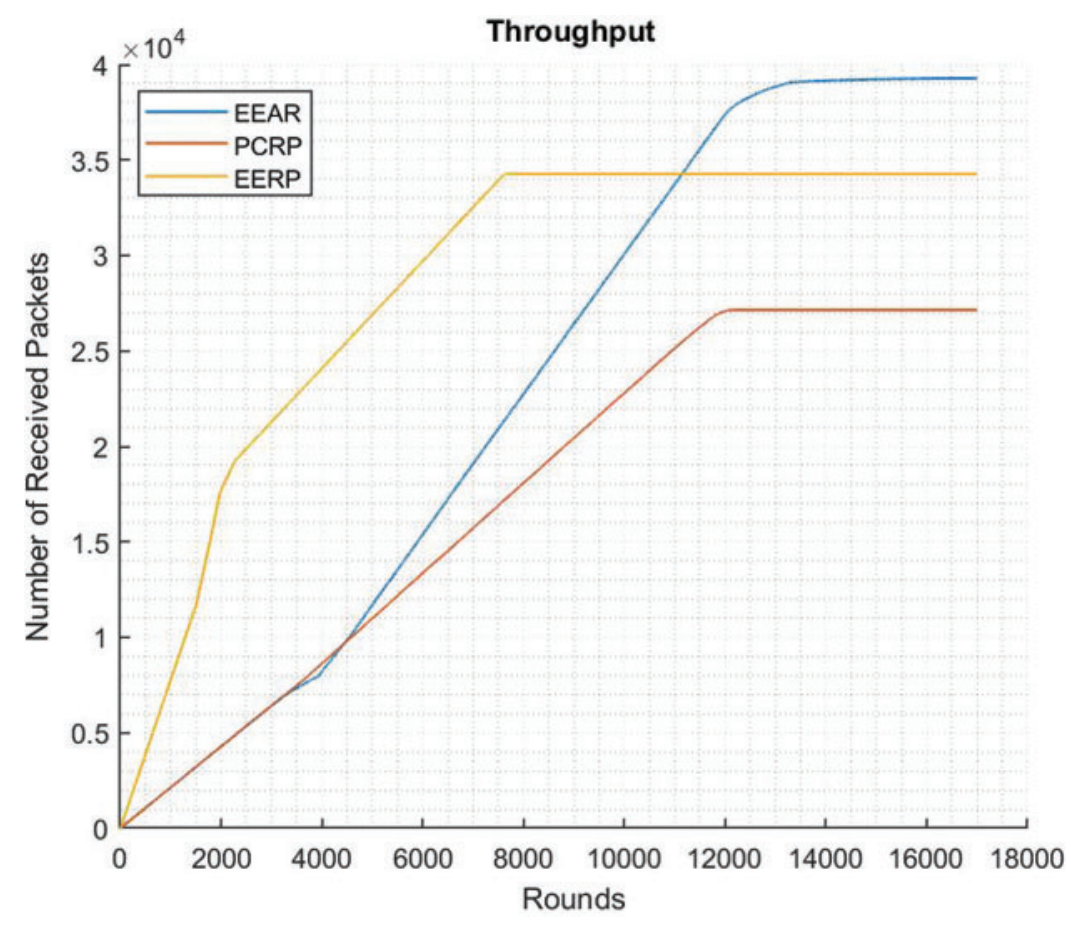

Figure 9: Analysis of throughput

\section{Conclusion and Future Enhancement}

Nowadays the wireless body area networks are widely used in many fields including healthcare, sport, entertainment, and the military. In healthcare, there is a significant application of WBANs that remotely serve the doctors and the patients. The doctors can monitor the medical situation of patients automatically, while the patients can follow the medical situation in their residences. Therefore, it is very important to enable efficient utilization and sustainable operation of their resources.

Indeed, the sensor nodes have a limited battery capacity that cannot be replaced or periodically recharged. In our study, we proposed an Efficient Energy Aware Routing mechanism (EEAR) to overcome the previous limitation. EEAR constructs the PSF to choose an optimum next hop node and develops the aggregation and filtration technique. Moreover, EEAR implements the hybrid harvesting technique to ensure sustainable energy. Many experiments are conducted and the comparisons of EEAR with PCRP and EERP are performed. The results proved that the EEAR is the most suitable routing mechanism for WBANs due to extending the network lifetime, balancing network traffic load, and reducing energy consumption.

The routing design of WBAN needs further enhancement at present; therefore, the following new research directions are recommended that are closely related to energy efficiency: 
- Develop the mechanism to be suitable for the dynamic movement of the body as the mobility has a further impact on the energy consumption.

- Design a lightweight method capable to deal with the memory of the node in an efficient way during aggregation and filtration of the packet.

- Add other parameters related to energy consumption to the cost function leading to the selection of the efficient next hop node.

Funding Statement: The authors received no specific funding for this study.

Conflicts of Interest: The authors declare that they have no conflicts of interest to report regarding the present study.

\section{References}

[1] S. Al-Janabi, I. Al-Shourbaji, M. Shojafar and S. Shamshirband, "Survey of main challenges (security and privacy) in wireless body area networks for healthcare applications," Egyptian Informatics Journal, vol. 18, no. 2, pp. 113-122, 2017.

[2] M. Ghamari, B. Janko, R. S. Sherratt, W. Harwin, R. Piechockic et al., "A survey on wireless body area networks for ehealthcare systems in residential environments," Sensors, vol. 16, no. 6, pp. 831, 2016.

[3] H. Fengye, L. Xiaolan, S. Meiqi, S. Dan and W. Liheng, "Wireless energy and information transfer in WBAN: An overview," IEEE Network, vol. 31, no. 3, pp. 90-96, 2017.

[4] T. Wu, F. Wu, J.-M. Redoute and M. R. Yuce, "An autonomous wireless body area network implementation towards IoT connected healthcare applications," IEEE Access, vol. 5, pp. 11413-11422, 2017.

[5] Y. Qu, G. Zheng, H. Ma, X. Wang, B. Ji et al., "A survey of routing protocols in wban for healthcare applications," Sensors, vol. 19, no. 7, pp. 1638, 2019.

[6] T. Hayajneh, G. Almashaqbeh, S. Ullah and A. V. Vasilakos, "A survey of wireless technologies coexistence in WBAN: Analysis and open research issues," Wireless Networks, vol. 20, no. 8, pp. 2165-2199, 2014.

[7] Z. Ullah, I. Ahmed, F. A. Khan, M. Asif, M. Nawaz et al., "Energy-efficient harvested-aware clustering and cooperative routing protocol for wban (e-harp)," IEEE Access, vol. 7, pp. 100036-100050, 2019.

[8] N. Javaid, A. Ahmad, Q. Nadeem, M. Imran and N. Haider, "iM-SIMPLE: iMproved stable increasedthroughput multi-hop link efficient routing protocol for wireless body area networks," Computers in Human Behavior, vol. 51, no. 4, pp. 1003-1011, 2015.

[9] A. Umare and P. Ghare, "Optimization of routing algorithm for wban using genetic approach," in Proc. 9th Int. Conf. on Computing, Communication and Networking Technologies, Bengaluru, India, pp. 1-6, 2018.

[10] F. Ullah, Z. Ullah, S. Ahmad, I. U. Islam, S. U. Rehman et al., "Traffic priority based delay-aware and energy efficient path allocation rout-ing protocol for wireless body area network," Journal of Ambient Intelligence and Humanized Computing, vol. 10, no. 10, pp. 3775-3794, 2019.

[11] K. M. Awan, N. Ashraf, M. Q. Saleem, O. E. Sheta, K. N. Qureshi et al., "A priority-based congestionavoidance routing protocol using iot-based heterogeneous medical sensors for energy efficiency in healthcare wireless body area networks," International Journal of Distributed Sensor Networks, vol. 15, no. 6, pp. 1-16, 2019.

[12] Y. Qu, G. Zheng, H. Wu, B. Ji and H. Ma, "An energy-efficient routing pro-tocol for reliable data transmission in wireless body area networks," Sensors, vol. 19, no. 19, pp. 4238, 2019.

[13] R. A. Khan, K. H. Mohammadani, A. A. Soomro, J. Hussain, S. Khan et al., "An energy efficient routing protocol for wireless body area sensor networks," Wireless Personal Communications, vol. 99, no. 4, pp. 1443-1454, 2018. 
[14] M. Anwar, A. H. Abdullah, A. Altameem, K. N. Qureshi, F. Masud et al., "Green communication for wireless body area networks: Energy aware link efficient routing approach," Sensors, vol. 18, no. 10, pp. 3237, 2018.

[15] V. C. Kumar, S. S. Prakash and S. Balandin, "Performance enhancement ofoptimized link state routing protocol for health care applications in wireless body area networks," in Proc. 23rd Conf. of Open Innovations Association (FRUCT), Bologna, Italy, pp. 195-203, 2018.

[16] B. Amin, N. Ullah, S. Ahmed, M. Taqi and A. Hanan, "Path-loss and energy efficient model (pleem) for wireless body area networks," in Proc. Int. Symp. on Wireless Systems and Networks, Lahore, Pakistan, pp. 1-6, 2017.

[17] M. Khan, Z. Ullah, A. Ahmed, B. Hayat, A. Almogren et al., "Energy harvested and cooperative enabled efficient routing protocol (ehcrp) for iot-wban," Sensors, vol. 20, no. 21, pp. 6267, 2020.

[18] W. R. Heinzelman, A. Chandrakasa and H. Balakrishnan, "Energy-efficient communication protocol for wireless microsensor networks," in Proc. 33rd Annual Hawaii Int. Conf. on System Sciences, Maui, HI, USA, pp. 1-10, 2000.

[19] O. A. Saraereh, A. Alsaraira, I. Khan and B. J. Choi, "A hybrid energy harvesting design for on-body Internet-of-Things (IOT) networks," Sensors, vol. 20, no. 2, pp. 407, 2020.

[20] A matter close to the heart: The right blood pressure levels. Zürich, Switzerland: Hirslanden, 2021. [Online]. Available: https://www.hirslanden.ch/en/corporate/topics-of-focus/heart-in-rhythm/blood-pressure.html.

[21] Single chip $2.4 \mathrm{GHz}$ transceiver product specification. Trondheim, Norway: Nordic Semiconductor, 2007. [Online]. Available: https://docplayer.net/334060-Nrf24101-single-chip-2-4ghz-transceiver-productspecification.html. 